\title{
A Minutiae-based Fingerprint Matching Algorithm Using Phase Correlation
}

\author{
Weiping Chen and Yongsheng Gao \\ School of Engineering, Faculty of Engineering and Information Technology, Griffith University, Australia \\ weiping.chen@student.griffith.edu.au, yongsheng.gao@griffith.edu.au
}

\begin{abstract}
Minutiae-based method is the most popular approach in fingerprint matching. However, most existing methods need to search for the best correspondence of minutiae pairs or use reference points (core and delta points) to estimate the alignment parameters. The problem of lost minutiae or spurious minutiae always occurs during the minutiae detection process. Hence, the corresponding pairs or reference points may not be found under this condition. This paper proposes a new minutiae-based fingerprint matching algorithm using phase correlation. We define a new representation called Minutiae Direction Map $(M D M)$. First, we convert minutiae sets into $2 D$ image spaces. Then the transformation parameters are calculated using phase correlation between two MDMs to align two fingerprints to be matched. The similarity of two fingerprints is determined by the distance between two minutiae sets. Our approach does not need to search for the corresponding minutiae pairs. Experimental results show that the proposed approach performed well in matching fingerprint minutiae sets, which greatly improved the economy of storage space.
\end{abstract}

\section{Introduction}

Fingerprint has been used as a method of personal identification for over a century. It is widely used in biometric authentication at present because of its uniqueness and permanence. A fingerprint consists of ridges and valleys. There are two basic features used in fingerprint recognition, i.e. ridge endings and ridge bifurcations. Other features are also used. According to features used in fingerprint recognition, automatic fingerprint recognition techniques are classified into minutiae-based, image-based and ridge feature-based approaches [1]. Ridge feature-based approach [2] is used when minutiae are difficult to extract in very lowquality fingerprint images, whereas other features of the fingerprint ridge pattern (e.g., local orientation and frequency, ridge shape, texture information) may be extracted more reliably than minutiae, even though their distinctiveness is generally lower.

Image-based approaches [3, 4] use the entire gray scale fingerprint images as a template to match against input fingerprint images. This approach needs a large size of storage space and fingerprint images are illegal to be stored in some nations.

Minutiae-based approach attempts to get the similarity degree between two minutiae sets. However, minutiae-based methods may make the computation more sophisticated and need to search for the best correspondence of minutiae pairs or ridge pairs [5] or use core or delta minutiae point to estimate the alignment [6]. The problem of lost minutiae or false minutiae always occurs during the minutiae detection process. Hence, the corresponding pairs may not be found under this condition.

In this paper, a new representation called Minutiae Direction Map (MDM) is introduced, which is generated by converting minutiae point sets into 2D image spaces. The alignment parameters are calculated using phase correlation between the input MDM and the template MDM. Our approach does not need to search for the corresponding minutiae pairs between the two fingerprints. The alignment parameters are obtained directly through phase correlation between two MDMs. Phase correlation [7] method provides straightforward estimation of rigid translation between two images. It was applied in image-based fingerprint recognition [3, 4]. However, fingerprint recognition is widely applied in embedded system or portable device which requires a small storage space. Since imagebased algorithms process entire fingerprint images, their large storage requirement for all fingerprint images limits their applicability. The proposed minutiae-based approach, stores merely a small number of minutiae points, which greatly reduces the storage requirement.

The paper is organized as follows: Section 2 gives the definition of phase correlation. Section 3 describes a fingerprint recognition algorithm using phase correlation, which includes the proposed representation 
MDM. Section 4 presents the experiment for evaluating matching performance and preliminary results. Conclusion is drawn in Section 5.

\section{Phase Correlation}

The phase correlation (PC) method is a popular choice for image registration because of its robust performance and computational simplicity [8]. It is based on the well-known Fourier shift theorem. Suppose two images $f_{1}$ and $f_{2}$, which differ only by a translation $d x$ and $d y$. The relationship between these two images is given by

$$
f_{2}(x, y)=f_{1}(x-d x, y-d y)
$$

Their corresponding Fourier Transforms $F_{1}$ and $F_{2}$ are related by

$$
F_{2}(u, v)=e^{-j 2 \pi(u d x / M+v d y / N)} F_{1}(u, v) .
$$

In other words, the Fourier magnitudes of the two images are same while their phases are different. This phase difference is directly related to displacement. The cross-phase spectrum (or normalized cross-phase spectrum) $P(u, v)$ which is represented by

$$
P(u, v)=\frac{F_{1}^{*}(u, v) F_{2}(u, v)}{\left|F_{1}^{*}(u, v) F_{2}(u, v)\right|}=e^{-j 2 \pi(u d m / M+v d n / N)}
$$

where $F_{1}^{*}(u, v)$ denotes the complex conjugate of $F_{1}(u, v)$. The 2D inverse Fourier transform of crossphase spectrum is given by

$$
p(m, n)=\frac{1}{M N} \sum_{u, v} P(u, v) e^{j 2 \pi(u m / M+v n / N)} .
$$

$p(m, n)$ is a delta function. The displacement coordinates are determined according to the location of the peak in the inverse cross-phase spectrum space.

\section{Fingerprint Matching Algorithm Using Phase Correlation}

In this section, we present the proposed the fingerprint matching algorithm using phase correlation based on minutiae points. Minutiae are prominent local ridge characteristics in fingerprint (see Figure 1). Our matching algorithm includes two stages: alignment stage and matching stage. In alignment stage, transformations including rotation and translation between two minutiae sets are calculated and then the input minutia set is aligned to models for similarity measurement. In this study, we assume there is no scaling different between two fingerprints as they are usually taken at the same resolution. The similarity between aligned input minutiae set and the template minutiae set is calculated in matching stage.
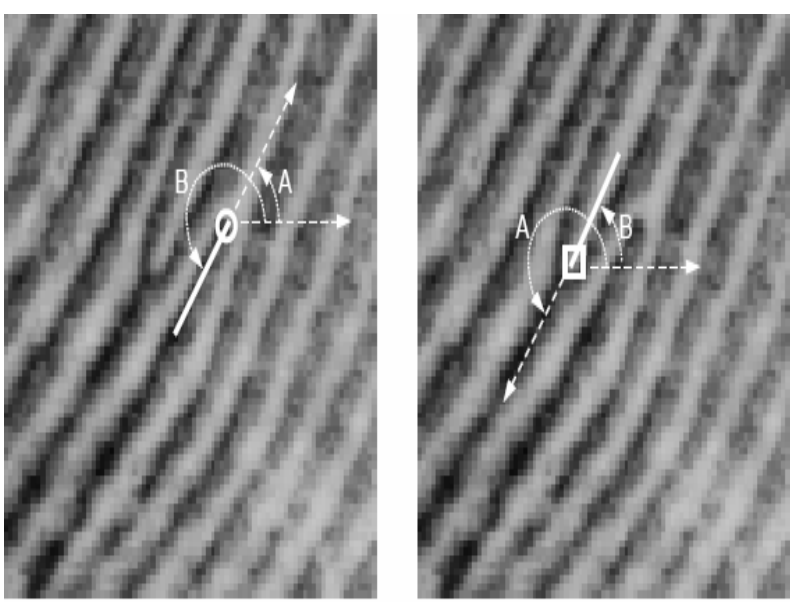

Figure 1. Two types of minutiae: ridge ending(left) and ridge bifurcation (right) [9].

\subsection{The Proposed Representation: Minutiae Direction Map}

Phase correlation can not be used to align two point sets directly. We present a new representation called Minutiae Direction Map (MDM) which is generated by converting minutiae point sets into a $2 \mathrm{D}$ image space. Alignment parameters are determined using phase correlation between two MDMs.

Let $M=\left(\left(x_{1}, y_{1}, \alpha_{1}\right), \ldots,\left(x_{N}, y_{N}, \alpha_{N}\right)\right)$ denote the set of $N$ minutiae in a fingerprint image. The image size is $C \times R$ and $\left(x_{i}, y_{i}, \alpha_{i}\right)$ are the three features (spatial position and orientation) associated with the ith minutiae in set $M$. Define the MDM of set $M$ as $\mathfrak{M}^{M}(m, n), \quad m \in[0, C-1], \quad n \in[0, R-1]$. It contains the angles of minutiae directions at the positions of minutiae points and 0 otherwise, which is written as

$$
\mathfrak{M}^{M}(m, n)=\left\{\begin{array}{l}
\cos \alpha_{i}+j \sin \alpha_{i} \quad m=x_{i}, n=y_{i}, \\
0 \quad \text { otherwise }
\end{array}\right.
$$


Where $\left(x_{i}, y_{i}, \alpha_{i}\right) \in M$. The size of the MDM $\mathfrak{M}^{M}$ is the same as that of the fingerprint image.

\subsection{Alignment}

In this stage, alignment parameters (displacements and rotation) are calculated between the template fingerprint and the input fingerprint. We assume the scaling is constant because the images in most applications are acquired at the same resolution. After converting minutiae sets into MDMs, the alignment parameters are estimated using phase correlation.

Suppose the possible rotation angle is from $-\theta_{\text {max }}$ to $\theta_{\max }$ with a angle spacing $\Delta \theta$. To every $\theta \in\left[-\theta_{\max }, \theta_{\max }\right]$, the input minutiae set $I$ is rotated into a new input minutiae set $I_{k}^{r}$, then convert the rotated input minutiae set into an input MDM $\mathfrak{M}^{\mathrm{I}_{\mathrm{k}}^{\mathrm{r}}}$ (see Section 3.1). A set of input MDMs $\mathfrak{M}^{\mathrm{I}_{\mathrm{k}}^{\mathrm{r}}}$ is obtained where $k=0,1,2, \ldots, M ; M=2 \theta_{\max } / \Delta \theta$. The highest correlation peak value $v_{k}$ in kth inverse phase correlation space is calculated by

$$
\begin{aligned}
& \left(d x_{k}, d y_{k}\right)=\arg \max _{m, n}\left\{p_{k}(m, n)\right\} \\
& v_{k}\left(d x_{k}, d y_{k}\right)=p_{k}\left(d x_{k}, d y_{k}\right),
\end{aligned}
$$

where $p_{k}(m, n)$ is the inverse phase correlation between kth input MDM $\mathfrak{M}^{\mathrm{I}_{\mathrm{k}}^{\mathrm{r}}}$ and template MDM $\mathfrak{M}^{\mathrm{T}}$ and $k=0,1,2, \ldots, M ; M=2 \theta_{\text {max }} / \Delta \theta \cdot\left(d x_{k}, d y_{k}\right)$ is the coordinate of $v_{k}$. If the size of MDM is $C \times R$, then $m \in[0, C-1]$ and $n \in[0, R-1]$.

A set of highest correlation peak value $V=\left\{v_{0}\left(d x_{0}, d y_{0}\right), \ldots, v_{M}\left(d x_{M}, d y_{M}\right)\right\} \quad$ is thus obtained, where $v_{k}\left(d x_{k}, d y_{k}\right)$ is maximum inverse PC value in $V, k=0,1,2, \ldots, M ; M=2 \theta_{\max } / \Delta \theta$.

Displacement $(\Delta x, \Delta y)$ between two fingerprint images is the $\left(d x_{k}, d y_{k}\right)$ with maximal value of $v_{k}$ in set $V$ and rotation angle $\theta_{r}$ is corresponding to index $k$ with the maximal value of $v_{k}$. Transformation parameters are thus calculated using equations (7).

$$
\begin{aligned}
& \theta_{r}=k * \Delta \theta-\theta_{\max } \\
& \Delta x=d x_{k} \\
& \Delta y=d y_{k}
\end{aligned}
$$

\subsection{Matching}

The input minutiae set $(I)$ is aligned into new set $\left(I^{\prime}\right)$ based on the transformation parameters, which are calculated in section 3.2. The overlapping region is defined according to minutiae locations in aligned input $\left(I^{\prime}\right)$ and template minutiae sets $(T)$. Two minutiae subsets $T_{o}$ and $I_{o}^{\prime}$ are minutiae located in overlapping area in template minutiae set $(T)$ and aligned input set $\left(I^{\prime}\right)$ respectively.

Let $A\left(x^{A}, y^{A}, \alpha^{B}\right)$ and $B\left(x^{B}, y^{B}, \alpha^{B}\right)$ denote two minutiae points in template $\left(T_{o}\right)$ and aligned input set $\left(I_{o}^{\prime}\right)$ respectively. The distance between these two points is defined in equation (8).

$d(A, B)=\sqrt{\left(x^{A}-x^{B}\right)^{2}+\left(y^{A}-y^{B^{\prime}}\right)^{2}+f^{2}(\Delta \theta)}$,

where

$\Delta \theta=\left\{\begin{array}{l}\left|\alpha^{A}-\alpha^{B}\right| \quad \text { if }\left|\alpha^{A}-\alpha^{B}\right| \leq 180^{\circ} \\ 360^{0}-\left|\alpha^{A}-\alpha^{B}\right| \text { if } 180^{\circ}<\left|\alpha^{A}-\alpha^{B}\right| \leq 360^{\circ},\end{array}\right.$

and

$$
f(x)=W \tan \left(\frac{x}{2}\right)
$$

$W$ is a weight to be determined experimentally in section 4.1.

Let $T_{o}=\left(A_{1}, A_{2}, \ldots, A_{p}\right)$ and $I_{o}^{\prime}=\left(B_{1}, B_{2}, \ldots, B_{q}\right)$ denote two minutiae sets. The distance between $T_{o}$ and $I_{o}^{\prime}$ is calculated using equation (11) to measure the similarity between the template minutiae set $(T)$ and input minutiae set $(I)$.

where

$$
S\left(T_{o}, I_{o}^{\prime}\right)=\max \left(D\left(T_{o}, I_{o}^{\prime}\right), D\left(I_{o}^{\prime}, T_{o}\right)\right)
$$

$$
D\left(T_{o}, I_{o}^{\prime}\right)=\frac{1}{p} \sum_{A_{i} \in T_{o}} \min _{B_{j} \in I_{o}^{\prime}} d\left(A_{i}, B_{j}\right) .
$$

If the distance $\left(S\left(T_{o}, I_{o}^{\prime}\right)\right)$ between two minutiae sets is less than a threshold, we think two images come from the same finger; otherwise the two images come from different fingers. 


\section{Experiment and Preliminary Results}

In our experiment, we captured fingerprints using SecuGen Hamster III (see Figure 2). This scanner employs a high-performance and maintenance-free optical sensor. The size of captured fingerprint image is $260 \times 300$ (width $\times$ height). We created a fingerprint database containing 400 fingerprints, which consists of 100 fingers and 4 impressions per finger. Sample impressions are illustrated in Figure 3. Minutiae extraction algorithm [9] is applied to get minutiae sets for fingerprint images. Genuine matching scores and impostor matching scores were calculated using the same strategy as in [10]. In genuine test, each sample is matched against the remaining samples of the same finger to compute the False Non Match Rate (FNMR). In the impostor matching test, the first sample of each finger is matched against the first sample of the remaining fingers in database to compute the False Match Rate (FMR). The total number of genuine tests and impostor tests are 600 and 4950 respectively.

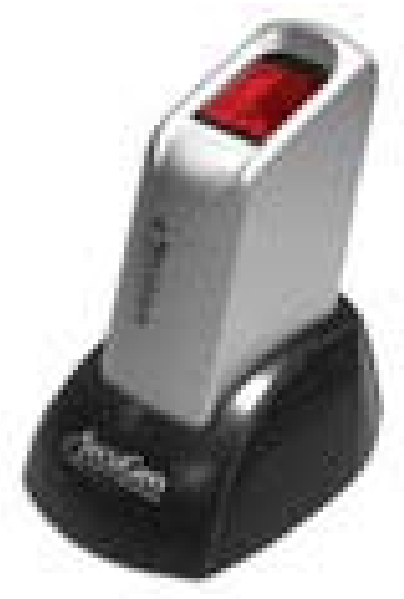

Figure 2. SecuGen® Hamster III fingerprint reader

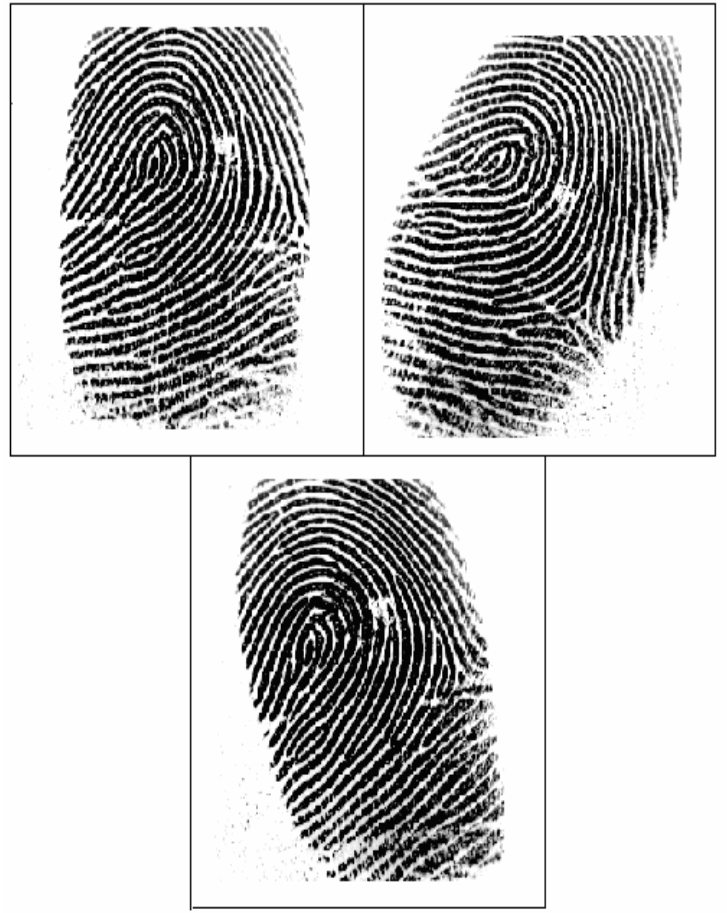

Figure 3. Example impressions of one finger.

\subsection{Determination of $W$}

The effect of $W$ in equation (10) was investigated using above database. Figure 4 shows the curve of equal error rate (EER) of FMR nd FNMR against the $W$. The EER decreases greatly from $W=0$ to $W=80$. Between $W=80$ and $W=200$, the EER is steady. Then it increases with the increase of $W$. For the following experiments, $W$ was set as 170 .

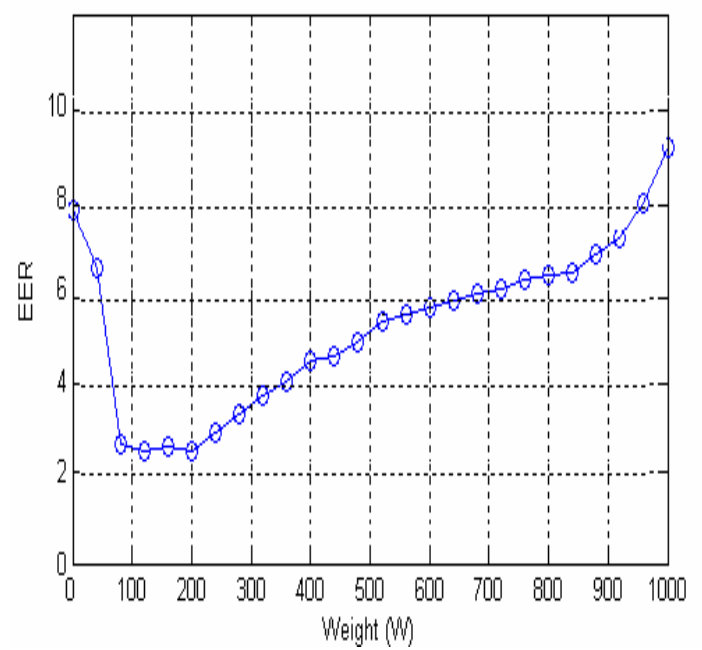

Figure 4. The effect of $W$ on the equal error rate (EER). 


\subsection{Preliminary Results}

In the experiment, Equal Error Rate (EER) was used to evaluate the system performance of the proposed method. In general, the performance of a matching algorithm can be depicted by the Equal Error Rate (EER), which is value where the FMR (False Match Rate) and FNMR (False Non-Match Rate) are equal [10]. FNMR and FMR are computed using equation (13).

$$
\begin{aligned}
& F M R(d)=\frac{\operatorname{card}\{\text { ims } \mid \text { ims } \geq d\}}{N I R A} \\
& \operatorname{FNMR}(d)=\frac{\operatorname{card}\{g m s \mid g m s<d\}}{N G R A}
\end{aligned}
$$

where gms and ims are genuine matching score and impostor matching score respectively. $d$ is the threshold of matching score. card denotes the cardinality of the given set. NGRA is the number of genuine matching attempts and NIRA is the number of impostor matching attempts. Given a threshold $d, F M R(d)$ and $F N M R(d)$ denote the percentage of $i m s \geq d$ and the percentage of gms $<d$ respectively [11].

Figure 5 shows the ROC curve in log-log scales for the proposed algorithm. The EER of the proposed algorithm is $2.44 \%$.

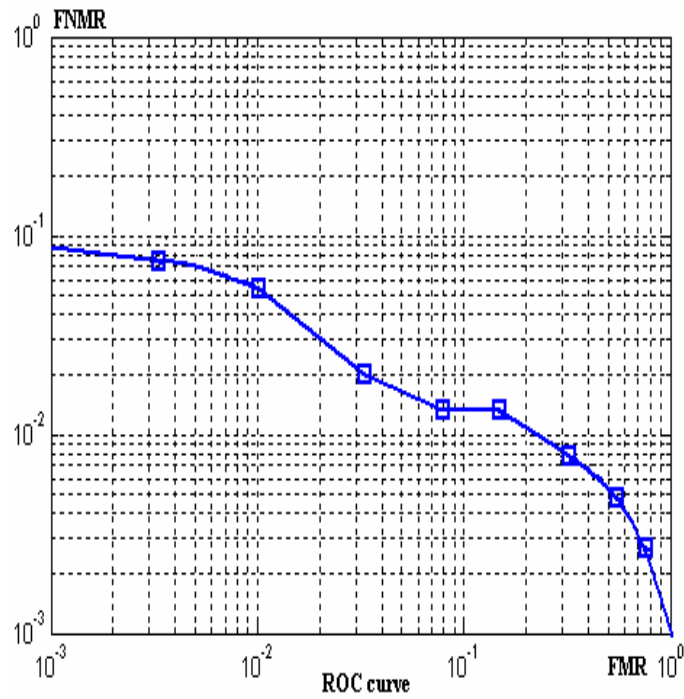

Figure 5. ROC curves of the proposed algorithm.

\section{Conclusion}

This paper proposes a novel minutiae-based fingerprint matching approach, which utilizes phase correlation to calculate the alignment parameters between two minutiae sets and the similarity is measured between the template minutiae set and the aligned input set. The computation of this proposed method is simple without the need of searching for corresponding minutiae pairs. In our algorithm, we only use the locations and directions of sparse minutiae points in fingerprints, which greatly reduces the storage space, comparing to current phase-based fingerprint matching techniques [3,4]. Experimental results show that the proposed approach performed well in matching fingerprint minutiae sets, which greatly improved the economy of storage space. As the FVC2004 fingerprint database contains partial impression, we will solve the incomplete/partial fingerprint problem in future research in order to make a direct performance comparison between the proposed method and those reported methods in [10].

\section{References}

[1] D.Maltoni, D.Maio, A.K.Jain, and S.Prabhakar, Handbook of Fingerprint Recognition. New York: Springer, 2003.

[2] A.K.Jain, S.Prabhakar, H.Lin, and S.Pankanti, "Filterbank-based fingerprint matching," IEEE Transactions on Image Processing, vol. 9, pp. 846-59, 2000.

[3] K.Ito, H.Nakajima, K.Kobayashi, T.Aoki, and T.Higuchi, "A Fingerprint Matching Algorithm Using Phase-Only Correlation," IEICE TRANS. FUNDAMENTALS, vol. E87-A, pp. 682-691, 2004.

[4] J.Zhang, Z.Ou, and H.Wei, "Fingerprint Matching Using Phase-Only Correlation and Fourier-Mellin Transforms," presented at The Sixth International Conference on Intelligent Systems Design and Applications (ISDA'06), 2006.

[5] A.K.Jain, H.Lin, and R.Bolle, "On-line fingerprint verification," IEEE Transactions on Pattern Analysis and Machine Intelligence, vol. 19, pp. 302-14, 1997.

[6] K.C.Chan, Y.S.Moon, and P.S.Cheng, "Fast fingerprint verification using subregions of fingerprint images," IEEE Transactions on Circuits and Systems for Video Technology, vol. 14, pp. 95-101, 2004.

[7] L.G.Brown, "A survey of image registration techniques," ACM Computing Surveys, vol. 24, pp. 325--376, 1992. 
[8] W. S. Hoge, "A subspace identification extension to the phase correlation method [MRI application]," IEEE Transactions on Medical Imaging, vol. 22, pp. 277-80, 2003.

[9] C.Watson and M. Garris, "User's guide to NIST Fingerprint image software2," National Institute of Standards and Technology, 2004.

[10] R. Cappelli, D. Maio, D. Maltoni, J. L. Wayman, and A. K. Jain, "Performance Evaluation of Fingerprint Verification Systems," IEEE Transactions on Pattern Analysis and Machine Intelligence, vol. 28, no. 1, January 2006.

[11] D. Maio, D. Maltoni, R. Cappelli, J. L. Wayman, and A. K. Jain, "FVC2000: Fingerprint Verification Competition," IEEE Transactions on Pattern Analysis and Machine Intelligence, vol. 24, no. 3, March 2002. 